\title{
Synthetic Control of Retinal Photochemistry and Photo- physics in Solution
}

\author{
Giovanni Bassolino ${ }^{\mathrm{a}}$, Tina Sovdat ${ }^{\mathrm{b}}$, Matz Liebel $^{\mathrm{a}}$, Christoph Schnedermann ${ }^{\mathrm{a}}$, Barbara Odell ${ }^{\mathrm{b}}$, \\ Timothy D.W. Claridge ${ }^{b}$, Philipp Kukura ${ }^{a *}$, Stephen P. Fletcher ${ }^{b *}$ \\ ${ }^{a}$ Department of Chemistry, Physical and Theoretical Chemistry Laboratory, University of Oxford, South Parks Road, \\ Oxford OX1 3QZ, U.K. \\ ${ }^{\mathrm{b}}$ Department of Chemistry, Chemistry Research Laboratory, University of Oxford, Mansfield Road, Oxford OXı 3TA, \\ U.K.
}

\begin{abstract}
Understanding how molecular structure and environment control energy flow in molecules is a requirement for the efficient design of tailor-made photochemistry. Here, we investigate the tunability of the photochemical and photophysical properties of the retinal protonated Schiff base chromophore in solution. Replacing the $n$-butyl amine Schiff base linkage, normally chosen to mimic the saturated linkage found in nature by aromatic amines results in the reproduction of the opsin shift and complete suppression of all isomerization channels. Modification of retinal by directed addition or removal of backbone substituents tunes the overall photoisomerization yield from o to 0.55 , the excited state lifetime from 0.4 to $7 \mathrm{ps}$ and activates previously inaccessible reaction channels to form 7-cis and 13-cis products. We observe a clear correlation between the presence of polarizable backbone substituents and photochemical reactivity. Structural changes that increase reaction speed were found to decrease quantum yields, and vice versa, so that excited state lifetime and efficiency are inversely correlated in contrast to the trends observed when comparing retinal photochemistry in protein and solution environments. Our results suggest a simple model where backbone modifications and Schiff base substituents control barrier heights on the excited state potential energy surface and thereby determine speed, product distribution and overall yield of the photochemical process.
\end{abstract}

Introduction. The retinal chromophore is ubiquitously used in nature to transform light into chemical, mechanical or electrical energy. ${ }^{.}$The desired function is invariably performed by a surrounding protein, but is initiated by an efficient photoisomerization of retinal covalently bound via a protonated Schiff base linkage. Although the choice of chromophore is convenient from a metabolic perspective, its reactivity, spectral overlap with the solar emission spectrum and photochemical specificity are poor for retinal and retinal protonated Schiff bases (RPSBs) in the absence of a protein environment. ${ }^{2-5}$ Remarkably, all of these parameters are dramatically improved in retinal-containing proteins, which is in line with the idea of optimized photobiological function. The discrepancy between solution and protein behavior has been largely attributed to the complexity of the protein pocket with its three-dimensional arrangement of amino acids resulting in a unique steric and dielectric environment. ${ }^{6,7}$

The tunability of absorption spectra, isomerization yield and reaction speed makes RPSB the ideal candidate for investigations aiming to unravel the molecular and structural origin of efficient photochemistry. ${ }^{6-15}$ As a consequence, RPSB has become a paradigm for understanding the origin of activation and suppression of ultrafast relaxation processes, which is essential for the rational engineering of photoreactivity. Despite numerous efforts, however, the reactive properties of RPSB in solution have been largely resistant to synthetic or environmental control. Small variations in both quantum yields (QYs) and excited state lifetimes (ESLs) have been reported, ${ }^{16-20}$ but it has never been possible to mimic the features observed in opsin photochemistry. The differences in RPSB reactivity between solution and protein environment are most dramatically illustrated by the photochemical properties of the chromophore in the bacterial proton pump bacterioRhodopsin $(\mathrm{bR})^{21,22}$ and in solution ${ }^{2}$ (Table 1). The red shift of the absorption spectrum in the protein, the so-called "opsin shift", has been investigated in detail synthetically, spectroscopically and computationally., ${ }^{4,0,23-33}$ The currently accepted mechanism of colour tuning is that absorption maximum is determined by the dielectric environment of the retinal binding pocket. ${ }^{3-37}$

Addressing other aspects of opsin photochemistry is more complex and a widely adopted approach has been to prepare pigment analogues containing retinal derivatives with modified backbones. ${ }^{8,38-42}$ Taken together, these studies point toward protein-induced steric and electrostatic interactions as a primary determinant in the outcome of the photoreaction..$^{43-47}$ To the best of our knowledge only one study on the photochemistry of a 
RPSB with a modified retinal backbone (11-cis-13demethyl RPSB) in solution has been reported. ${ }^{48}$ In our recent exploratory work ${ }^{49}$ we demonstrated that addition of methyl to the 10-position of the retinal backbone dramatically affects RPSB solution photochemistry, reducing the ESL by almost one order of magnitude from 4 ps to 0.55 ps toward the lifetime observed in bR (o.5 ps). Together with the changes in ESL, the modification caused a $50 \%$ decrease in the isomerization yield, suggesting a inverse correlation between reaction speed and yield, but could only provide an incomplete picture as to the mechanistic origin of the observed effect.

TABLE 1. Retinal protonated Schiff base photochemistry in protein and solution.

\begin{tabular}{ccc}
\hline Lys & $n-\mathrm{Bu}$ \\
abs $\lambda_{\max }(\mathrm{nm})$ & 568 & 446 \\
Excited State Lifetime (ps) & 0.5 & 4.3 \\
Product (Quantum Yield) & 13-cis(0.64) & 11-cis(0.16) \\
9-cis $(0.05)$
\end{tabular}

In this work, we thus systematically explored the influence of both backbone and Schiff base substitution on RPSB photochemistry. By appropriate choice of the Schiff base, we succeeded in reproducing the shift of absorption maximum observed for RPSBs in the protein environment, with the tail of the absorption reaching all the way to the near-infrared (70o nm). Addition or removal of polarizable substituents from the retinal backbone for the natural $n$-butyl $(n \mathrm{Bu})$ Schiff base allowed us to tune the ESL from 400 fs to $7 \mathrm{ps}$, and achieve spread of the overall reaction yield from effectively o to 0.55 . We further induced changes in the selectivity of these reactions - either completely closing secondary isomerization pathways, or obtaining 7-cis or 13-cis isomers rather than the 9-cis isomer observed with natural RPSB in solution (see the Supporting Information, Figure $\mathrm{SF} 18)$. Our results demonstrate that remarkable tunability of RPSB photochemistry can be achieved in solution, without a specific spatial, steric and dielectric environment as provided by a protein pocket evolved through natural selection.

\section{Materials and Methods}

Synthesis. We used the general synthetic approach depicted in Scheme 1 to prepare a library of retinal derivatives (Table 2). A series of consecutive HornerWadsworth-Emmons (HWE) reactions and DIBAL-H reductions were employed to prepare the modified retinals (2b-2m) (Scheme 1). ${ }^{12}$ Substituents at $C_{10}$ of the backbone (derivatives $2 \mathrm{~b}-\mathrm{f}, \mathrm{2l}$ ) were introduced via coupling of commercially available $\beta$-Ionone with the ap-

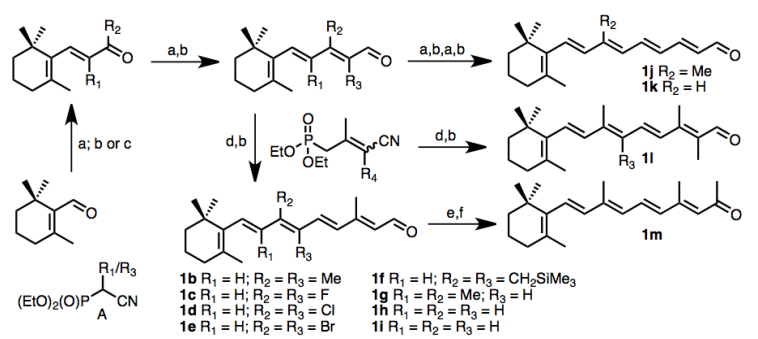

Scheme 1. Synthesis of modified retinal chromophores. a) BuLi, A; b) DIBALH; c) MeLi; d) BuLi; e) MeLi; f) TPAP, NMO

propriate phosphonate $\mathbf{A}^{50,51}$ The HWE reaction inevitably generated a mixture of $E / Z$-isomers. However, subsequent reduction to the aldehyde allowed for the isolation of the desired isomer in all cases. The chain was then extended with (E/Z)-diethyl (3-cyano-2methylallyl)phosphonate; $;^{50,52,53}$ again generating a mixture of isomers. Upon reduction, all-trans $\mathrm{C}_{10}$ substituted retinals were isolated (2b-f). The methyl group at position 14 of the retinal backbone $(\mathbf{2 k}, \mathbf{2 l})$ was installed using a methylated modification of (E/Z)-diethyl (3cyano-2-methylallyl)phosphonate ${ }^{52}$ prepared from chloroacetone and diethyl(cyanoethyl)phosphonate. Methylation of $\mathrm{C}_{8}(\mathbf{2 g})$ was implemented through HWE coupling of $\beta$-cyclocitral with diethyl(cyanoethyl)phosphonate. The methyl group at $\mathrm{C}_{9}$ was introduced via addition of MeLi to the resulting nitrile. ${ }^{54}$ The polyene chain was extended in a manner analogous to the $\mathrm{C}_{10}$ substituted retinal synthesis. Addition of methyl group to $C_{15}(\mathbf{2 m})$ was achieved through quantitative 1,2-addition of MeLi to commercially available all- $E$ retinal $^{55}$ followed by oxidation with catalytic TPAP, which was found to be a more suitable oxidizing agent than $\mathrm{MnO}_{2}$ or Dess-Martin periodinane oxidations.

TABLE 2. Chain substituents for the backbone modified retinals.

\begin{tabular}{ccccccc}
\hline Compound & X8 & X9 & X10 & X13 & X14 & X15 \\
\hline NAT (1a) & H & Me & H & Me & H & H \\
10Me (1b) & $\mathrm{H}$ & $\mathrm{Me}$ & $\mathrm{Me}$ & $\mathrm{Me}$ & $\mathrm{H}$ & $\mathrm{H}$ \\
10F (1c) & $\mathrm{H}$ & $\mathrm{Me}$ & $\mathrm{F}$ & $\mathrm{Me}$ & $\mathrm{H}$ & $\mathrm{H}$ \\
10Cl (1d) & $\mathrm{H}$ & $\mathrm{Me}$ & $\mathrm{Cl}$ & $\mathrm{Me}$ & $\mathrm{H}$ & $\mathrm{H}$ \\
$10 \mathrm{Br}(1 \mathrm{e})$ & $\mathrm{H}$ & $\mathrm{Me}$ & $\mathrm{Br}$ & $\mathrm{Me}$ & $\mathrm{H}$ & $\mathrm{H}$ \\
$10 \mathrm{Si}(1 \mathrm{f})$ & $\mathrm{H}$ & $\mathrm{Me}$ & $\mathrm{CH}_{2} \mathrm{Si}\left(\mathrm{CH}_{3}\right)_{3}$ & $\mathrm{Me}$ & $\mathrm{H}$ & $\mathrm{H}$ \\
$\mathbf{8 M e}(1 \mathrm{~g})$ & $\mathrm{Me}$ & $\mathrm{Me}$ & $\mathrm{H}$ & $\mathrm{Me}$ & $\mathrm{H}$ & $\mathrm{H}$ \\
9-dm (1h) & $\mathrm{H}$ & $\mathrm{H}$ & $\mathrm{H}$ & $\mathrm{Me}$ & $\mathrm{H}$ & $\mathrm{H}$ \\
13-dm (1i) & $\mathrm{H}$ & $\mathrm{Me}$ & $\mathrm{H}$ & $\mathrm{H}$ & $\mathrm{H}$ & $\mathrm{H}$ \\
9,13-dm (1j) & $\mathrm{H}$ & $\mathrm{H}$ & $\mathrm{H}$ & $\mathrm{H}$ & $\mathrm{H}$ & $\mathrm{H}$ \\
14Me (1k) & $\mathrm{H}$ & $\mathrm{Me}$ & $\mathrm{H}$ & $\mathrm{Me}$ & $\mathrm{Me}$ & $\mathrm{H}$ \\
10,14-Me (1l) & $\mathrm{H}$ & $\mathrm{Me}$ & $\mathrm{Me}$ & $\mathrm{Me}$ & $\mathrm{Me}$ & $\mathrm{H}$ \\
15Me (1m) & $\mathrm{H}$ & $\mathrm{Me}$ & $\mathrm{H}$ & $\mathrm{Me}$ & $\mathrm{H}$ & $\mathrm{Me}$
\end{tabular}



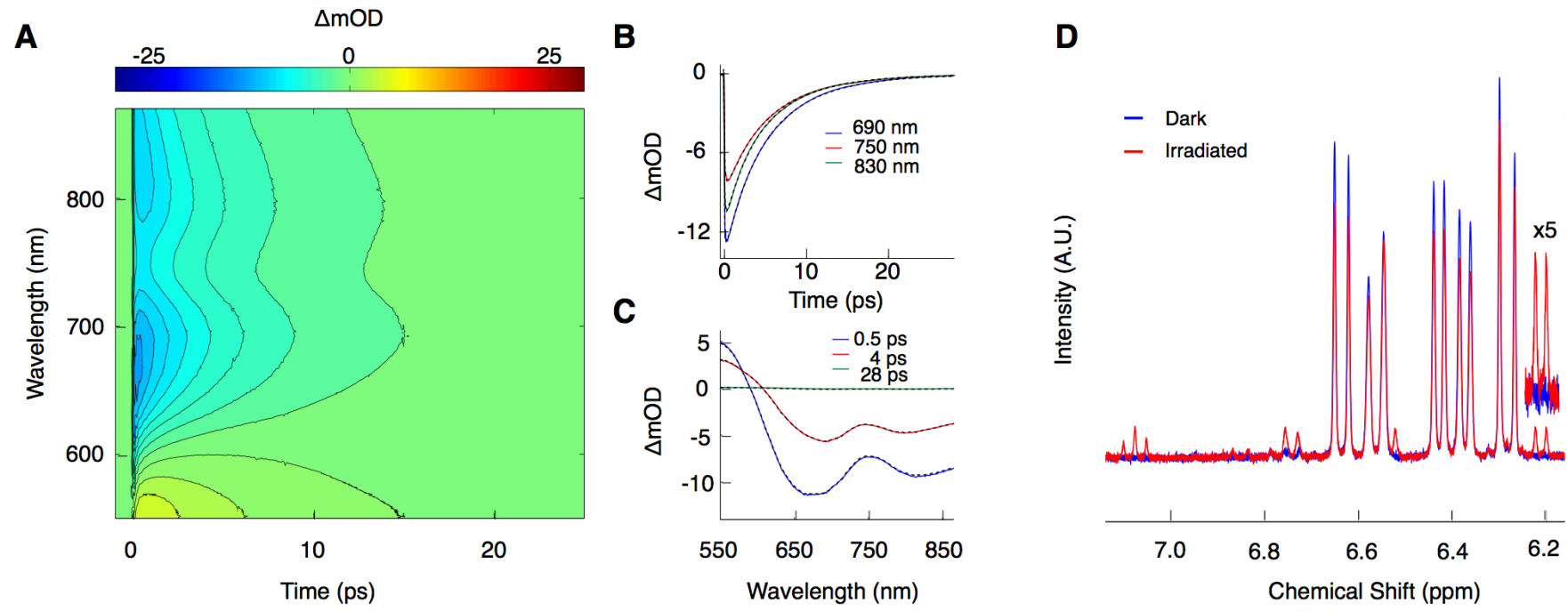

Figure 1. Experimental approach to determine RPSB photochemistry. (A) Differential absorbance map as a function of time delay and probe wavelength recorded for natural $n$ Bu-RPSB (1a). (B) Time traces at selected probe wavelengths throughout the stimulated emission band. (C) Transient absorption spectra corresponding to the temporal evolution of the data. (D) Typical ${ }^{1} \mathrm{H}$ NMR spectra before and after irradiation of natural $n B u-R P S B$.

Demethylated retinal analogues (2h-j) were accessed by stepwise formation of additional double bonds from the ring towards the aldehyde functional group, so that demethylation at $\mathrm{C}_{9}(\mathbf{2 h})$ was obtained through two consecutive cycles of HWE coupling followed by DIBAL reduction starting from $\beta$-cyclocitral. Likewise, $\mathrm{C}_{13}$ methyl group removal (2i) employs an analogous strategy starting from the appropriate aldehyde.

Schiff base formation was achieved by condensing a retinal (2a-2m) with the appropriate amine. Chromophores containing aliphatic amines were prepared by stirring the two components for an hour at $0{ }^{\circ} \mathrm{C}$ over dry $4 \AA$ Å molecular sieves in methanol under rigorously dark conditions. Imines with aromatic amine moieties were prepared at room temperature with extended reaction times (10-12 h). Formation of protonated Schiff bases (1a-m and 3-5, Figure 2) was achieved via the addition of a drop of neat trifluoroacetic acid (TFA) to a solution of the imine in methanol. ${ }^{56}$ All photosensitive materials were handled under dim red light illumination. See the Supporting Information for details of the preparation, purification and characterization of each derivative.

Ultrafast spectroscopy. Laser pulses were delivered by a LightConversion PHAROS-6W amplifier (1030 nm, $180 \mathrm{fs}, 5.5 \mathrm{~W}$ at $10 \mathrm{kHz}$ ). $15 \mathrm{~mW}$ of the output was used for white light generation in sapphire to generate broadband probe pulses. The remainder was used for second harmonic generation (SHG) in $\mathrm{BBO}$ followed by sum frequency generation (SFG) between second harmonic and fundamental (BBO).$^{57}$ The obtained output at $343 \mathrm{~nm}(500 \mathrm{~mW})$ pumped a white-light seeded 2- stage non-collinear optical parametric amplifier NOPA ${ }^{58}$ to generate the excitation pulse ( $500 \mathrm{~nm}, 25 \mathrm{fs}, 30 \mathrm{~mW})$. The pump was compressed using chirped mirrors (Layertec) and characterized with SHG frequency-resolved optical gating (FROG) ${ }^{59}$ in a $10 \mu \mathrm{m}$ BBO. A flow cell with path length of $500 \mu \mathrm{m}$ and $120 \mu \mathrm{m}$ windows was used for the experiments. The sample flow was adjusted to replenish the sample between consecutive pulses. The diameter of the pump and probe beams were measured to be $150 \mu \mathrm{m}$ and $50 \mu \mathrm{m}$, with the pulse energies adjusted to 20 and 2 nJ, respectively. Broadband detection was performed by a home built single shot prism spectrograph $^{60}$ equipped with a LW-ELIS-1024A-1394 detector. We corrected the differential absorbance maps for probe chirp from solvent only traces to generate transient absorption traces as a function of probe wavelength and time delay (Figure $1 \mathrm{~A}$ ) and then fitted the latter via a global optimization routine. ${ }^{61}$ All transient absorption data analysis was performed in MATLAB. ${ }^{62}$ The dynamics of all compounds could be described by a triexponential function convolved with a gaussian instrument response and a step function (Figure $1 B$ ). Typical transient differential absorption spectra are shown in Figure 1C. The three time constants ranged from o.2-0.4 ps, 0.53 ps and 3-10 ps for the first, second and third constant, respectively (see the Supporting Information, Table S1 and Figures $\mathrm{SF}_{1}-\mathrm{SF}_{17}$ for details on each compound). The coherent response due to the overlap of the pump and probe pulse is modelled with a combination of gaussian derivatives up to the ninth order. 
Determining the excited state lifetime. The differential absorption between 550 and $850 \mathrm{~nm}$ was used to evaluate the average lifetime for each RPSB. From the global optimization routine, each time constant was retrieved together with a decay associated spectrum (DAS) ${ }^{61}$ For all detection wavelengths within the stimulated emission (SE) band $(\sim 600-850 \mathrm{~nm})$ the average ESL was evaluated as a weighted average of the decay constants and the absolute value of the DAS amplitude, according to the following formula:

$$
\mathrm{ES} L_{\lambda}=\frac{\Sigma_{k} \tau_{k}\left|D A \mathrm{~S}_{k \lambda}\right|}{\Sigma_{k}\left|D A \mathrm{~S}_{k \lambda}\right|}
$$

The values reported in the results section are the average of the lifetime values obtained along the SE band (see the Supporting Information, Table $\mathrm{S} 1$ for details on each compound).

Quantum yield measurements. A CW diodepumped solid state laser $(532 \mathrm{~nm})$ was used to irradiate a glass test tube containing $1 \mathrm{~mL}$ of a stirred $\sim 3 \mathrm{mM}$ (OD>>2) solution of RPSB in $\mathrm{CD}_{3} \mathrm{OD}$. Expanding the beam to a diameter of $0.8 \mathrm{~cm}$ at an incident power of 0.2 $\mathrm{mW}$ resulted in irradiation in the linear regime. Experiments were performed in such conditions to ensure less than $10 \%$ conversion of the starting material, so that irradiation times ranged between 15 and 40 minutes depending on the isomerization yield. The amount of photoproduct formed was determined by $500 \mathrm{MHz}$ or $700 \mathrm{MHz}{ }^{1} \mathrm{H}$ NMR spectroscopy by integration, comparing the average of several starting material signals with the average of several product signals (Figure 1D). Sample concentration was determined by adding $10 \mu \mathrm{L}$ of a $1 \% \mathrm{v} / \mathrm{v}$ solution of an appropriate internal standard to $700 \mu \mathrm{L}$ of non-irradiated RPSB and comparing the average area of the RPSBs signals with the area of the internal standard ones in the ${ }^{1} \mathrm{H}$ NMR spectrum (see the Supporting Information Table S2 for details on the internal standards used for each compound).

For the aromatic amine containing compounds (3-5, Figure 2), the QY and excited state dynamics were measured in $\mathrm{CH}_{3} \mathrm{CN}$ or $\mathrm{CD}_{3} \mathrm{CN}$. For this class of compounds no isomerization product was detectable via ${ }^{1} \mathrm{H}$ NMR spectroscopy.

Photoproduct determination. Irradiations of the RPSBs were carried out as described above, adjusting the reaction time to achieve $\sim 20 \%$ conversion. Under these conditions no new species were formed as determined by ${ }^{1} \mathrm{H}$ NMR spectroscopy. Photoproducts were identified by $1 \mathrm{D}$ and/or $2 \mathrm{D}$ NOESY, TOCSY, and COSY experiments (see the Supporting Information). ${ }^{63}$ In some cases photoproducts were identified by comparison with a fully characterized synthetic standard (see the Supporting Information).
Computational Methods. Ground state optimized geometries for all molecules studied in this paper were obtained with Gaussian $09^{64}$ using the wb97xd/cc-pVDZ potential. ${ }^{65,66}$ As a test for the optimized structure to be a local minimum, the second derivatives of the Hessian matrix were checked to be positive. ${ }^{67}$ Merz-Kollman (MK) partial atomic charges ${ }^{68,69}$ for ground and first singlet excited state were evaluated with the wb97xd/6$31+G(d, p)^{65,70,71}$ potential at the ground state optimized geometry. The solvent (methanol) was treated at the CPCM level. ${ }^{72,73}$

\section{Results}

Aromatic Amines. To reproduce the "opsin shift", we used an aromatic amine to form the Schiff base, thus introducing additional electron delocalization to the RPSB system. Three compounds (3-5) exhibiting a broad range of photophysical behaviour, in addition to natural RPSB (1a), are shown in Figure 2. The red shift of the absorption when using aromatic rather than aliphatic amines can be seen in the UV-Vis absorption spectra (Figure $2 \mathrm{~B}$ ). It consisted of up to $90 \mathrm{~nm}$ (to $534 \mathrm{~nm}$ ) for $p$-phenilenediamine RPSB 5 relative to $n B u$ RPSB 1a. In addition, the spectra broadened considerably. In contrast to the uniform trend observed for shifts in the absorption maximum, the effect of the extended $\pi$-system on the ESLs (Figure $2 \mathrm{C}$ ) differed: In two cases - $p$ methoxy-aniline RPSB (4) and p-phenilenediamine RPSB (5) - the lifetime decreased compared to natural RPSB (1a) (3.4 and 1.2 ps vs 6.8 ps, respectively), while in the case of the unsubstituted aniline (3) it increased (12 ps).

A comparison of the transient spectra at 1 ps delay time for natural $n B$ RPSB (1a) and the three aromatic derivatives (3-5) is shown in Figure 2D. The features of the transient spectrum of natural nBu-RPSB (1a) are well known including a broad SE band (6oo->90o $\mathrm{nm}$ ) overlapping with an excited state absorption band (ESA) that results in a distinctive double peak shape. Another ESA band is present at $580 \mathrm{~nm}$, with the tail of the ground state bleach (GSB) visible up to $550 \mathrm{~nm} .{ }^{74}$ The most striking feature for the aromatic derivatives (3-5) is the separation and reduced spectral overlap of the red ESA and SE bands. As the absorption maxima for these derivatives is considerably red shifted, the GSB is now clearly visible in our observation window up to $\sim 600$ $\mathrm{nm}$. Remarkably, the isomerization yield for all RPSBs with aromatic Schiff bases (3-5) is zero. 
A
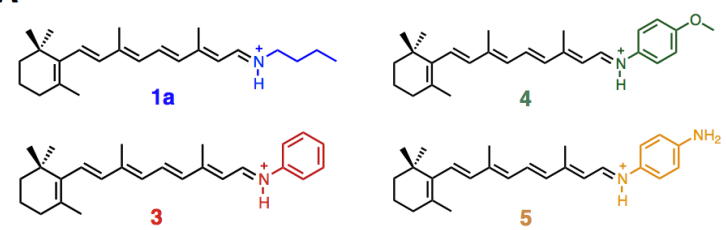

B

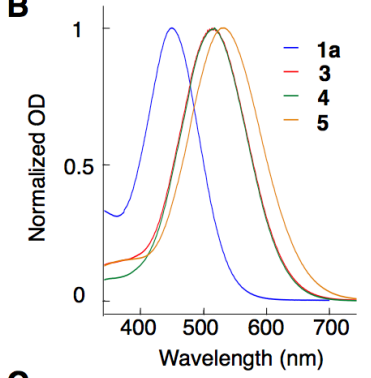

C

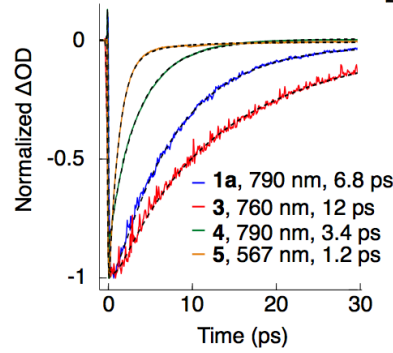

D

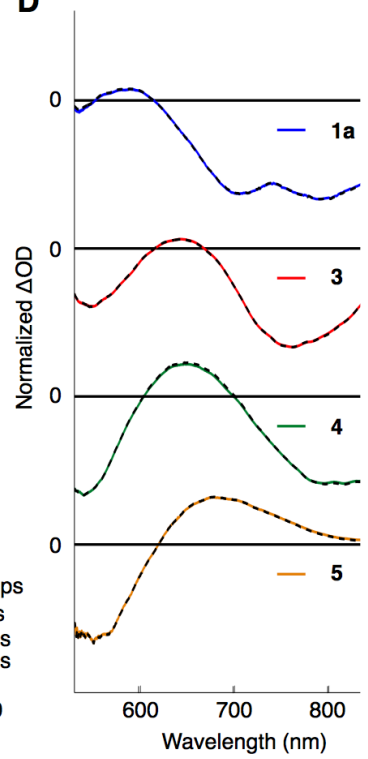

difference in electron density upon excitation. Based on this reasoning, we focused on two types of synthetic modifications: addition or removal of methyl groups from the natural backbone ( $2 \mathbf{b}$ and $\mathbf{2 g - \mathbf { m }}$ ), and addition of substituents with different electronic character at the C10 position (2b-f). The former class of compounds allowed us to probe the effect of the substitution position, while the latter helped us to understand the importance of the character of the substitution. Evaluation of the ground state MK partial atomic charges and the difference in MK charges upon excitation revealed a pattern for these new derivatives qualitatively similar to the one found for natural RPSB (1a), but differences were observed at the position of substitution (see the Supporting Information, Figures SF19-21 ).

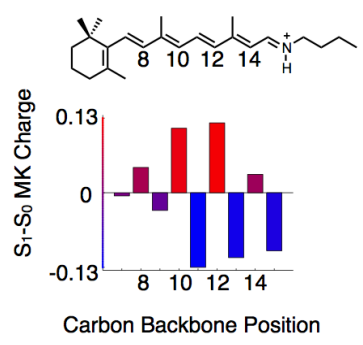

Figure 3. Difference in MK charges of the excited and ground states for polyene chain carbons of natural $n \mathrm{Bu}$ RPSB 1 .

Figure 2. Modification of the Schiff base. Comparison for RPSBs obtained with aromatic amines (A) structures, (B) absorption spectra, (C) decay kinetics, and (D) transient spectra at 1 ps after photoexcitation with a resonant $25 \mathrm{fs}$ pump pulse. Dashed lines represent the fits obtained from the global optimization routine.

Modification of the retinal backbone. While extending the $\pi$-system allowed us to reproduce the "opsin shift", it did not enable us to change the topography of the excited state potential energy surface (PES) and change the QY beyond completely halting photoisomerization. Prompted by published work reporting a considerable electron redistribution following excitation for RPSBs, ${ }^{75,76}$ we evaluated the difference in MK partial atomic charges between the first excited singlet state and the ground electronic state for natural $n \mathrm{Bu}$ RPSB (1a) (Figure 3). A positive sign of this charge difference corresponds to depletion of electron density, whilst a negative sign to an increase in electron density for the atom in question. One would thus expect the effect of backbone substitution to be greatest on the topography of the excited electronic state when there is a significant

The results of the photochemical investigation on the examined compounds are reported in Figure 4. A general trend for the effects of the performed substitutions on the photochemistry becomes immediately clear: all derivatives with substituents added to the backbone ( $\mathbf{1 b}$ $\mathbf{1 g}$ and $\mathbf{1 k}-\mathbf{m}$ ) exhibit faster excited state decay than natural $n \mathrm{Bu}$ RPSB $\mathbf{1 a}$, while demethylation (11h-j) increases the ESL. The only exception is 9-demethyl RPSB $\mathbf{1 h}$, which exhibits a slightly lower ESL than 1a. The ESL can be tuned over a range of values smaller than for the chromophore in bacterioRhodopsin (500 $\mathrm{fs}$ ), as in the case of 10,14-dimethyl RPSB $\mathbf{l l}$ (400 fs), to more than an order of magnitude larger than in bR, in the case of 9,13demethyl $\mathbf{1 j}$ (7 ps). Addition of substituents at the 10position always shortens the ESL, and the effect is correlated with the polarizability of halogenated substituents $(\mathrm{Br}>\mathrm{Cl}>\mathrm{F})$. A single demethylation (1i) extends the ESL, with removal of both methyl backbone groups (ij) having the largest effect in this sense. 


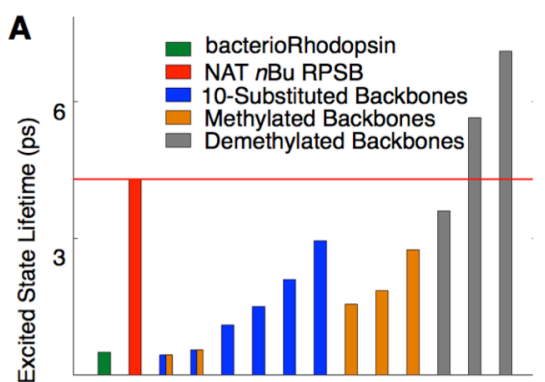

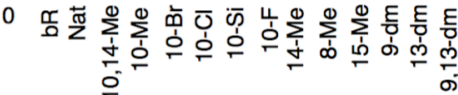

B

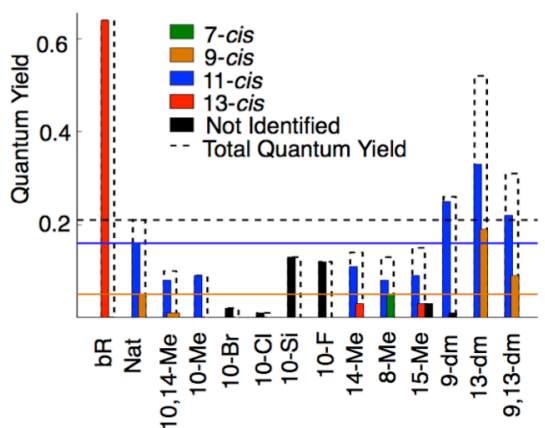

C
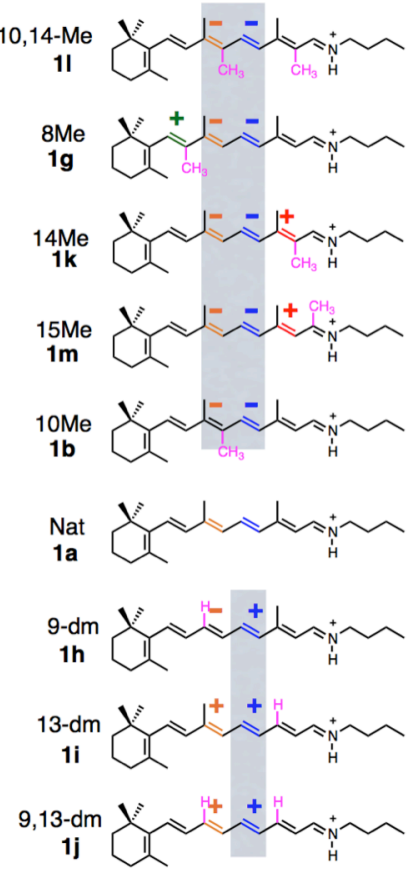

Figure 4. Kinetics and isomerization yields for backbone-modified RPSB derivatives in solution. (A) Excited state lifetimes, and (B) QY for photoproduct formation. The values for bR value are taken from references 21 and 22.(C) Structures of the (de)methylated all-trans isomers and changes to isomerization yields. Backbone modifications are shown in pink. Isomerizing bonds are coloured according to the graph in B. “+” or "-” signs refer to the change in quantum yield for the corresponding double bond with respect to 1 .

A similar trend is observed for the corresponding QY of isomerization (Figure $4 \mathrm{~B}$ ). Removal of backbone substituents ( $\mathbf{1 h}-\mathbf{j})$ increases the total isomerization yield,

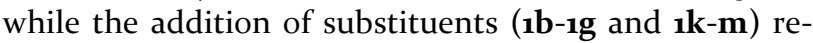
duces the yield. The tunability ranges from a barely detectable overall yield (<<0.02) for 10-chloro RPSB id to a total yield of 0.55 for the 13-demethyl isomer (1i). For those species where the photoproducts identities could be determined, the major photoproduct was the 11-cis isomer. Addition or removal of substituents in the 9- and 10-position ( $\mathbf{1 b}, \mathbf{1 c}, \mathbf{1 f})$ lead to an increased reaction selectivity, with yields as high as $25 \%$ in the case of the 9-demethyl RPSB (1h). In contrast, addition of methyl-groups to other $\mathrm{C}=\mathrm{C}$ bonds $(\mathbf{1 g}, \mathbf{1 k}, \mathbf{1 m})$ activated isomerization channels previously silent in the natural backbone 1a. Most notably, these included the activation of the $7,8 \mathrm{C}=\mathrm{C}$ isomerization upon methylation of the 8-position (19), and the activation of the 13,14 C=C channel upon methylation of the 14- and 15- positions (1k and $\mathbf{1 m}$ respectively). The latter is the only channel active in the isomerization of retinal in the bacteriorhodopsin proton pump, and this photoisomerization channel is undetectable in our measurements of prolonged irradiated natural $n B u$ RPSB (1a) (see the Supporting Information, Figure SF18). The changes in isomerization yield, illustrated as '+' for increasing or opening a photoisomerization channel, and '-' for decreasing or closing a pathway upon (de)methylation of the backbone, are summarized in Figure ${ }_{4} \mathrm{C}$.

\section{Discussion}

Aromatic Amines. Extension of the RPSB $\pi$-system by addition of an aromatic unit as the amine moiety (35) has two major effects on the absorption spectra, redshifting the absorption maximum by about $80 \mathrm{~nm}$, and allowing for limited tunability of the latter in the 520 $\mathrm{nm}$ region. This bathochromic shift is accompanied by changes in the ESL with no apparent trend. The presence of electron donating groups $(\mathbf{4}, \mathbf{5})$ reduces the ESL, while it increases in their absence (3) with respect to natural RPSB (1a). Independently of the effect on the ESL, all aromatic Schiff bases are completely unreactive.

To understand the origin of the complete lack of photochemical activity, it is useful to consider the change in the distribution of the positive charge upon excitation from the ground state to the first excited singlet state. ${ }^{75,76}$ For the natural $n \mathrm{Bu}$-RPSB, a partial translocation of the positive charge is observed predominantly toward the $\beta$-ionone ring increasing the single bond character of $\mathrm{C}=\mathrm{C}$ bonds, and vice versa. This dramatic change in equilibrium bond lengths upon excitation is well reflected in the ground state pre-resonance Raman spectra of RPSB, that are dominated by $C-C$ and $C=C$ stretching coordinates, ${ }^{77}$ as expected for strongly displaced modes (Figure $5 \mathrm{~A}) .^{78}$ Replacing the $n \mathrm{Bu}$ moiety with an aromatic one has only a negligible 
A
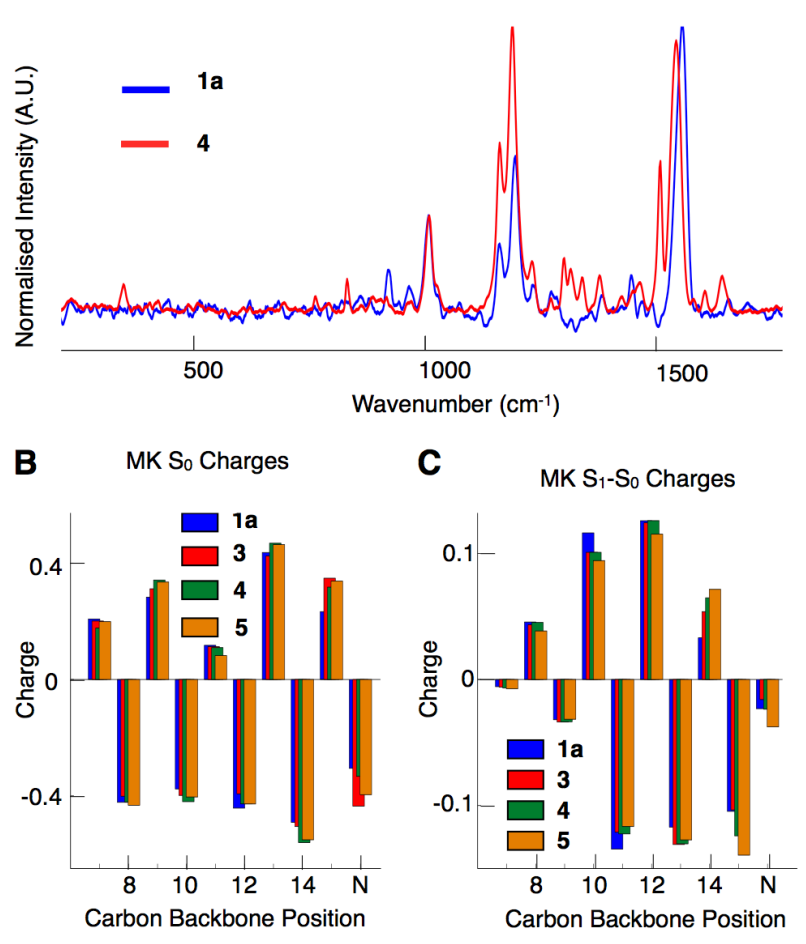

Figure 5. The electronic character in the Franck-Condon region is unchanged upon substitution of an aromatic Schiff base. (A) Pre-resonance Raman spectra of natural $n B u$ RPSB (1a) and p-methoxy-aniline RPSB (4). (B) MK charges for the ground electronic state, and (C) difference in MK charges between ground and first excited state computed for different protonated Schiff bases.

effect on the appearance of the Raman spectrum, except for a splitting of the $\mathrm{C}=\mathrm{C}$ stretching band $\left(1500 \mathrm{~cm}^{-1}\right.$ region) caused by symmetric and asymmetric combination of the backbone stretching with the Schiff base stretching modes (Figure $5 \mathrm{~A}$ ). Calculations of the MK charges for the optimized ground state geometry (Figure $\left.{ }_{5} \mathrm{~B}\right)$ and charge differences at the same point between first excited state and ground state (Figure ${ }_{5} \mathrm{C}$ ) show remarkable similarity independently of the Schiff base character.

These observations suggest that the nature of the excited electronic state in the Franck-Condon region, the character of the initial charge translocation, and the resulting changes in bond lengths are very similar irrespective of the nature of the Schiff base. The dramatic suppression of photochemical reactivity must have its origin outside the Franck-Condon region. A possibility is that the addition of the aromatic amine not only serves to extend the $\pi$-system but also to provide an alternative relaxation channel such as a Twisted Intramolecular Charge Transfer (TICT) ${ }^{79}$ state, from which the system can decay efficiently without backbone isomerization.
An alternative, but useful interpretation focuses on the conversion of photon into nuclear kinetic energy in the form of vibrational excitations. The latter are critical in determining the rate constants for the various relaxation channels and thus branching ratios for ultrafast decay processes that take place on timescales faster or comparable to vibrational relaxation times. Charge translocation necessarily causes dramatic changes in equilibrium bond lengths, which in turn causes the deposition of photon energy in nuclear coordinates that are structurally coupled to the movement of the charge. Our results suggest that the direction of energy flow is critical in determining the photochemical reactivity of retinal. If that energy flow can be influenced by electrostatic effects, charged amino acid residues in the protein pocket could play a similar role to the aromatic amines used in this work. However, in the protein the effect is likely opposite, pushing the charge even more towards the retinal backbone compared to a simple alkane as in natural $n B u$ RPSB (1a). Attempts to test this hypothesis in solution failed due to the low nucleophilicity of aromatic amines with strongly electron withdrawing groups, but this does provide exciting prospects for future work. Overall, controlling the direction of charge translocation and thus nuclear energy flow is a potential control mechanism for photochemical.

Backbone Modification. Although Schiff base modification allowed us to completely halt the photoisomerization of RPSB in solution, we could not investigate the molecular origin of photochemical reactivity and selectivity. Photoisomerization of natural all-trans $n B$ RPSB (1a) in solution produces a mixture of 11-cis and 9-cis RPSBs, with the former being dominant. In contrast, the same process in bR produces exclusively the 13-cis isomer with a three-fold higher overall yield. Comparison of Figures $4 \mathrm{~A}$ and $4 \mathrm{~B}$ shows a clear correlation between ESL and isomerization yield. In contrast to the impression created from the comparison of RPSB dynamics in proteins (bR) and in solution, which suggests that reaction speed is a prerequisite for efficient photochemistry, these results clearly demonstrate an opposite trend.

In addition, the observed specific activation and deactivation of isomerization channels depended on the position of backbone substitutions. Within the range of the species studied here, we have observed a wide variety of selectivity profiles, and induced isomerization for all backbone $C=C$ double bonds. In some cases we have observed almost complete specificity (for example, 10methyl RPSB (1b) and 9-demethyl RPSB (1h)), in other cases the overall quantum yield approaches the one observed in the bR protein environment ( 0.55 for $\mathbf{1 i}$ vs 0.65 for $b R$ ). The observed trends qualitatively agree with a simple model based on polarizability, charge stabilization and concomitant effects on barrier heights resulting in different branching ratios (Figure 6). 
A

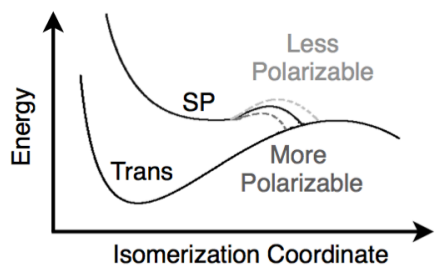

B

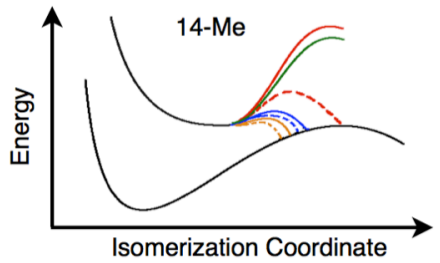

C
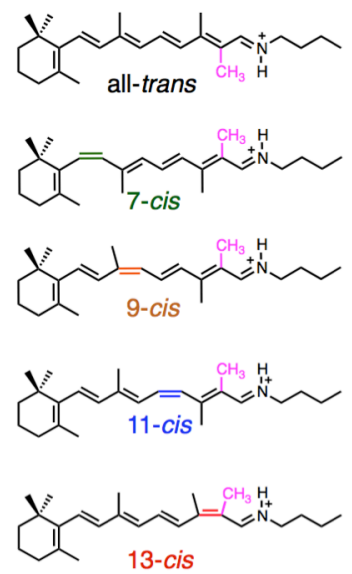

D

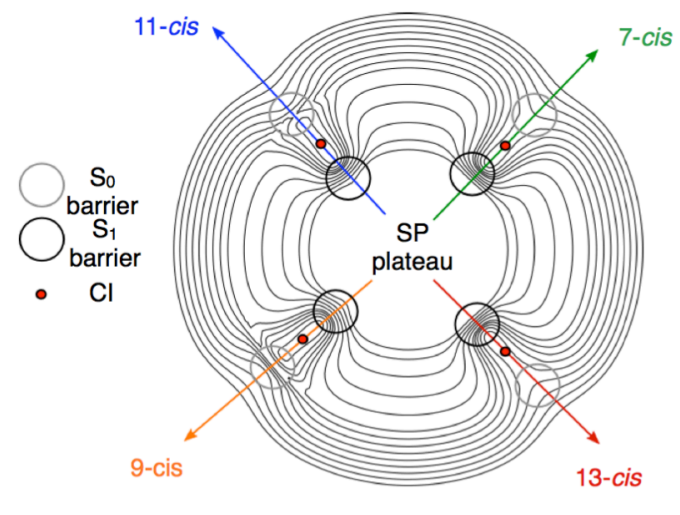

Figure 6. Modification of excited state barrier heights as a mechanism for tuning RPSB photochemistry. (A) General effect on the addition of polarizable groups to the retinal backbone on excited state barriers and the location of the surface crossing. (B) Effect of addition of a methyl group to the 14-position on the barriers towards excited state decay using distortions towards the 7, 9, 11 and 13-cis products (green, orange, blue and red, respectively). (C). Structure for all-trans 14-methyl RPSB (1k) and the possible photoproducts. Isomerizing bonds are the colored ones. (D) Multi-dimensional representation of the excited state PES indicating all major decay pathways with differing barrier heights.

The general trend in ESL suggests a simplified potential energy surface as shown in Figure 6A: addition of polarizable groups decreases the ESL and vice versa. The effects of change in substituent polarizability are shown as dashed lines, using solid lines to qualitatively depict the situation in natural $n B u$ RPSB (1a). Given that the lifetime is likely controlled by a barrier in the excited electronic state toward internal conversion, ${ }^{2,15,19,80-83}$ it is reasonable to assume that the effect of the polarizable substituents is to lower that barrier. The correlation between ESL and isomerization yield suggests this to be a consequence of the barrier lowering. This observation is in line with isomerization coordinates at least partially contributing to excited state decay, and a lower barrier leading to an earlier crossing along the isomerization coordinate which in turn results in lower isomerization yield. Such early crossing agrees with experiments on bacteriorhodopsin that showed how the initial stationary point reached in the excited electronic state, after relaxation out of the Franck-Condon region, corresponds to only little evolution along the isomerization coordinate. ${ }^{84}$ Additional support for this hypothesis has been recently provided by high-level quantum chemical calculations that observed the existence of a barrier on the excited electronic state on the path towards the conical intersection $(\mathrm{CI}) .^{15,83}$ Furthermore, the connection between internal conversion and isomerization coordinate is in agreement with the symmetries of any vibrational modes coupling the two electronic states of different symmetry, i.e. a requirement for non-totally symmetric modes, such as backbone torsions and hydrogen wags. Models that provide a mean toward excited state decay by distortion of the backbone require that, at least in principle, all $\mathrm{C}=\mathrm{C}$ bonds can mediate internal conversion and thus appear as potential photoproducts. The product distribution would then depend on the relative barrier heights and crossing points and could therefore be altered from the naturally occurring pathways (9-cis and 11-cis) by changing the barrier heights by backbone substitution.

As an example, consider 14-methyl RPSB (1k), a substitution that results in the complete suppression of the 9-cis channel, reduction of 11-cis yield and the activation of the 13-cis channel (Figure 6B). All of these observations are in line with the simple model introduced above. Methylation in general causes all barriers to drop, lowering the quantum yields of the naturally occurring channels (Figure 6A). In the case of 14-methyl RPSB (1k), 9-cis as a product becomes undetectable (yellow, Figure 6B), while the 11-cis yield drops from 0.16 to 0.11 (blue). For both the 9-cis and the 11-cis channels, the lowering of the excited state barrier leads to faster decay, but a lower yield as the surface crossing occurs earlier along the isomerization coordinate. At the same time the 13-cis channel is activated (QY of 0.03 ) as a result of lowering of the barrier along the 13-cis isomerization channel sufficiently to generate a detectable amount of photoproduct (red channel). The $C_{7}=C_{8}$ double bond, however, being too distant from the point of substitution, experiences only negligible changes to the excited state barrier height, remaining inactive. We remark that exactly the same line of argument can be used to rationalise all changes in QYs observed in this work. 8-methyl RPSB (19), for example, acts in the same way as 14-methyl RPSB (1k), except that now the nearby $\mathrm{C}_{7}=\mathrm{C}_{8}$ double bond is activated while the 9-cis product becomes undetectable and 11-cis drops. Removal of methyl groups, instead, raises the barriers for all inactive 
channels to such a degree that they remain inactive, while a higher barrier, even though reducing the competitivity of the channel, leads to a later CI which increases the quantum yield for the naturally active pathways (9-cis and 11-cis).

To visualize such a highly-dimensional PES, it is convenient to project the individual isomerization coordinates onto non-orthogonal dimensions (Figure 6C, D). These coordinates do not represent normal modes of the molecules but simply the definition of the PES in degrees of freedom related to a backbone isomerization. Although it may appear counterintuitive, in this representation, the system evolves along all coordinates simultaneously from the stationary point without requiring a bifurcation of the potential energy surface near the Franck-Condon region. Taken together, all of these evolutions represent the overall structural change taking place during possible isomerization reactions, as opposed to the more common backbone stretching or torsional degrees of freedom. At this stage, the barrier heights determine the possible decay rates as well as the likelihood of isomerization through the location of the crossing point as discussed for 14-methyl case (1k) above (Figure 6B).

Although such a model suggests multi-exponential decay with component amplitudes related to the isomerization yield for each particular decay channel, their experimental observation by transient absorption spectroscopy is likely to be challenging. Although in principle additional, lower amplitude components could be added, features such as vibrational cooling make the quantification of more than three decay constants challenging as addition of more parameters tends to improves the fit so that determining a clear cut-off point is difficult. Further testing of the proposed model would thus require time-resolved vibrational spectroscopy with high sensitivity, where the growth of individual photoproducts can be observed and differentiated.

A conceptually simple, but challenging (in terms of thermal stability of the RPSB) method to obtaining high QY would be to incorporate a strongly electron withdrawing Schiff base that pushes the charge towards the retinal backbone maximizing the activation of backbone-twisting decay channels and thus the isomerization yield. Combining such efforts with backbone modifications such as a 9-demethyl or 10-demethyl RPSB (1h, 1b respectively) that show remarkable selectivity, provides scope for the artificial design of materials with high isomerization yields and specificity to an extent that was thought to exist only in an evolutionoptimized, three-dimensional protein environment.

\section{Conclusions}

Our overall model of charge stabilization, either by the Schiff base or by addition of polarizable groups to the backbone, suggests a simple but powerful mechanism that allows tuning of photochemical reactivity by rational, synthetic design. The general methodology of synthetic tuning and spectroscopic characterization was applied to retinal isomerization due to the extensive experimental and theoretical literature precedent. Applications to energy or electron transfer for other chromophores found in evolution optimized protein environments will be equally possible in the future.

\section{ASSOCIATED CONTENT}

Supporting Information. Synthetic procedure and characterization data for all the compounds, details of the analysis on the transient absorption data, QY measurement and MK charge analysis. This material is available free of charge via the Internet at http://pubs.acs.org

\section{Author Information}

\section{Corresponding author}

philipp.kukura@chem.ox.ac.uk stephen.fletcher@chem.ox.ac.uk

\section{Funding}

The EPSRC supports P.K. and S.P.F. by Career Acceleration Fellowships (EP/Hoo3541/1 \& EP/Hoo3711/1) and this research project through standard grant EP/Koo663o/1 awarded to P.K. and S.P.F.

\section{Notes}

The authors declare no competing financial interest.

\section{ACKNOWLEDGEMENT}

We are immensely grateful to Giulio Cerullo, Eberhard Riedle and Nicolaus Ernsting and their research roups for assistance in the construction of the ultrafast setup, and to Christopher D.P. Duffy and Vincent Liégeois for helpful discussions. The authors would like to acknowledge the use of the University of Oxford Advanced Research Computing (ARC) facility in carrying out this work.

\section{ABBREVIATIONS}

RPSB, retinal protonated Schiff base; $n B u, n$-butylamine; bR, bacterioRhodopsin; QY, quantum yield; HWE, HornerWadsworth-Emmons; SHG, second harmonic generation; SFG, sum frequency generation; DAS, decay associated spectrum; ESL, excited state lifetime; MK, Merz-Kollman; SE, stimulated emission; ESA, excited state absorption; GSB, ground state bleach; PES, potential energy surface; TICT, twisted intramolecular charge transfer; SP, stationary point; $\mathrm{CI}$, conical intersection.

\section{REFERENCES}

(1) Spudich, J.; Yang, C.; Jung, K.; Spudich, E. Annu. Rev. Cell Dev. Biol. 20oo, 16, 365 .

(2) Freedman, K. A.; Becker, R. S. J. Am. Chem. Soc. 1986, 108,1245

(3) Waddell, W. H.; Crouch, R.; Nakanishi, K.; Turro, N. J. J. Am. Chem. Soc. 1976, 98, 4189.

(4) Blatz, P. E.; Liebman, P. A. Exp. Eye Res. 1973, 17, 573

(5) Fukada, Y.; Okano, T.; Shichida, Y.; Yoshizawa, T.; Trehan, A.; Mead, D.; Denny, M.; Asato, A. E.; Liu, R. S. H. Biochemistry 1990, 29, 3133. 
(6) Kochendoerfer, G.; Lin, S.; Sakmar, T.; Mathies, R. Trends Biochem. Sci. 1999, 24, 300.

(7) Verhoeven, M.; Bovee-Geurts, P.; de Groot, H.; Lugtenburg, J.; DeGrip, W. J. Mol. Biol. 20o6, 363, 98.

(8) Ottolenghi, M.; Sheves, M. J. Membr. Biol. 1989, 112, 193.

(9) Becker, R. S. Photochem. Photobiol. 1988, 48, 369.

(10) Wanko, M.; Hoffmann, M.; Strodel, P.; Koslowski, A.; Thiel, W.; Neese, F.; Frauenheim, T.; Elstner, M. J. Phys. Chem. B 2005, 109, 3606 .

(11) Mathies, R.; Lin, S.; Ames, J.; Pollard, W. Annu. Rev. Biophys. and Biophys. Chem. 1991, 20, 491.

(12) Dawadi, P.; Lugtenburg, J. Molecules 2010, 15, 1825.

(13) Altoe, P.; Cembran, A.; Olivucci, M.; Garavelli, M. Proc. Natl. Acad. Sci. U. S. A. 2010, 107, 20172.

(14) Warshel, A. Nature 1976, 26o, 679.

(15) Valsson, O.; Filippi, C. J. Chem. Theory Comput. 2o10, $6,1275$.

(16) Waddell, W. H.; Hopkins, D. L.; Uemura, M.; West, J. L. J. Am. Chem. Soc. 1978, 100, 1970.

(17) Waddell, W. H.; West, J. L. J. Phys. Chem. 1980, 84, 134 .

(18) Blatz, P. E.; Mohler, J. H.; Navangul, H. V. Biochemistry 1972, 11, 848.

(19) Zgrablic, G.; Voitchovsky, K.; Kindermann, M.; Haacke, S.; Chergui, M. Biophys. J. 2005, 88, 2779.

(20) Bismuth, O.; Friedman, N.; Sheves, M.; Ruhman, S. J. Phys. Chem. B 2007, 111, 2327.

(21) Mathies, R. A.; Brito Cruz, C. H.; Pollard, W. T.; Shank, C. V. Science 1988, 240, 777

(22) Tittor, J.; Oesterhelt, D. FEBS Lett. 1990, 263, 269

(23) Yan, B.; Spudich, J. L.; Mazur, P.; Vunnam, S.; Derguini, F.; Nakanishi, K. J. Biol. Chem. 1995, 270, 29668.

(24) Sheves, M.; Kohne, B.; Mazur, Y. J. Chem. Soc. Chem. Commun. 1983, 1232.

(25) Houjou, H.; Inoue, Y.; Sakurai, M. J. Phys. Chem. B 2001, $105,867$.

(26) Baloghnair, V.; Carriker, J. D.; Honig, B.; Kamat, V.; Motto, M. G.; Nakanishi, K.; Sen, R.; Sheves, M.; Tanis, M. A.; Tsujimoto, K. Photochem. Photobiol. 1981, 33, 483.

(27) Kakitani, H.; Kakitani, T.; Rodman, H.; Honig, B. Photochem. Photobiol. 1985, 41, 471.

(28) Sakmar, T. P.; Franke, R. R.; Khorana, H. G. Proc. Natl. Acad. Sci. U. S. A. 1991, 88, 3079.

(29) Neitz, M.; Neitz, J.; Jacobs, G. H. Science 1991, 252, 971.

(30) Chan, T.; Lee, M.; Sakmar, T. P. J. Biol. Chem. 1992, 267, 9478.

(31) Rajput, J.; Rahbek, D.; Andersen, L.; Hirshfeld, A.; Sheves, M.; Altoe, P.; Orlandi, G.; Garavelli, M. Angew. Chem. Int. Ed. 2010, 49, 1790.

(32) Sekharan, S.; Sugihara, M.; Buss, V. Angew. Chem. Int. Ed. $2007,46,269$.

(33) Rajamani, R.; Lin, Y.; Gao, J. J. Comput. Chem. 2o11, 32,854 .

(34) Wanko, M.; Hoffmann, M.; Frauenheim, T.; Elstner, M. J. Phys. Chem. B, 2008, 112, 11462.

(35) Sudo, Y.; Yuasa, Y.; Shibata, J.; Suzuki, D.; Homma, M. J. Biol. Chem. 2011, 286, 11328.

(36) Melaccio, F.; Ferre, N.; Olivucci, M. Phys. Chem. Chem. Phys. 2012, 14, 12485.

(37) Wang, W.; Nossoni, Z.; Berbasova, T.; Watson, C.; Yapici, I.; Lee, K.; Vasileiou, C.; Geiger, J.; Borhan, B. Science 2012, 338, 1340.

(38) Corson, D.; Crouch, R. Photochem. Photobiol. 1996, 63, 595 .

(39) Baasov, T.; Sheves, M. J. Am. Chem. Soc. 1985, 107, 7524 .
(40) Drachev, L. A.; Drachev, A. L.; Chekulaeva, L. N.; Evstigneeva, R. P.; Kaulen, A. D.; Khitrina, L. V.; Khodonov, A. A.; Lazarova, Z. R.; Mitsner, B. I. Arch. Biochem. Biophys. 1989, 270, 184 .

(41) Liu, R. S. H.; Asato, A. E. In Chemistry and biology of synthetic retinoids; Dawson, M. I., Okamura, W. H., Eds.; CRC Press: Boca Raton, 1990, p 51-75.

(42) Balogh-Nair, V.; Nakanishi, K. In Chemistry and biology of synthetic retinoids; Dawson, M. I., Okamura, W. H., Eds.; CRC Press: Boca Raton, 1990, p 147-176.

(43) Schiffmiller, R.; Callender, R. H.; Waddell, W. H.; Govindjee, R.; Ebrey, T. G.; Kakitani, H.; Honig, B.; Nakanishi, K. Photochem. Photobiol. 1985, 41, 563

(44) deGrip, W.; deLange, F.; Bovee, P.; Verdegem, P.; Lugtenburg, J. Pure Appl. Chem. 1997, 69, 2091.

(45) Derguini, F.; Nakanishi, K. Photobiochem. Photobiophys. 1986, 13, 259.

(46) Sheves, M.; Ottolenghi, M. In Chemistry and biology of synthetic retinoids; Dawson, M. I., Okamura, W. H., Eds.; CRC Press: Boca Raton, 1990, p 99-123.

(47) Crouch, R. K. In Chemistry and biology of synthetic retinoids; Dawson, M. I., Okamura, W. H., Eds.; CRC Press: Boca Raton, 1990, p 125-146.

(48) Becker, R. S.; Freedman, K. J. Am. Chem. Soc. 1985, 107,1477

(49) Sovdat, T.; Bassolino, G.; Liebel, M.; Schnedermann, C.; Fletcher, S. P.; Kukura, P. J. Am. Chem. Soc. 2012, 134, 8318.

(50) DeLange, F.; Bovee-Geurts, P.; VanOostrum, J.; Portier, M.; Verdegem, P.; Lugtenburg, J.; DeGrip, W. Biochemistry 1998, 37, 1411.

(51) Iorga, B.; Ricard, L.; Savignac, P. J. Chem. Soc., Perkin Trans. 1 2000, 3311.

(52) Creemers, A.; Lugtenburg, J. J. Am. Chem. Soc. 2002, 124,6324 .

(53) Trehan, A.; Mirzadegan, T.; Liu, R. S. H. Tetrahedron 1990, 46, 3769 .

(54) Barakat, A.; Brenna, E.; Fuganti, C.; Serra, S. Tetrahedron-Asymmetry 20o8, 19, 2316.

(55) Arens, J. F.; Van Dorp, D. A. Nature 1946, 158, 622.

(56) Delera, A. R.; Torrado, A.; Iglesias, B.; Lopez, S. Tetrahedron Lett. 1992, 33, 6205.

(57) Homann, C.; Schriever, C.; Baum, P.; Riedle, E. Opt. Express 2008, 16, 5746.

(58) Cerullo, G.; De Silvestri, S. Rev. Sci. Instrum. 2003, 74, 1.

(59) Kane, D. J.; Trebino, R. IEEE J. Quantum Electron. 1993, 29, 571 .

(6o) Megerle, U.; Pugliesi, I.; Schriever, C.; Sailer, C. F.; Riedle, E. Appl. Phys. B: Lasers Opt. 2oo9, 96, 215.

(61) Shrager, R.; Hendler, R. J. Phys. Chem. B 2003, 107, 1708.

(62) ; The Mathworks inc.: Natick, MA, 2012.

(63) Claridge, T. D. W. High-Resolution NMR Techniques in Organic Chemistry; 2nd Ed. ed.; Elsevier Science: Oxford, 2008.

(64) Gaussian 09, Revision A.o2, Frisch, M. J.; Trucks, G. W.; Schlegel, H. B.; Scuseria, G. E.; Robb, M. A.; Cheeseman, J. R.; Scalmani, G.; Barone, V.; Mennucci, B.; Petersson, G. A.; Nakatsuji, H.; Caricato, M.; Li, X.; Hratchian, H. P.; Izmaylov, A. F.; Bloino, J.; Zheng, G.; Sonnenberg, J. L.; Hada, M.; Ehara, M.; Toyota, K.; Fukuda, R.; Hasegawa, J.; Ishida, M.; Nakajima, T.; Honda, Y.; Kitao, O.; Nakai, H.; Vreven, T.; Montgomery, J. A., Jr.; Peralta, J. E.; Ogliaro, F.; Bearpark, M.; Heyd, J. J.; Brothers, E.; Kudin, K. N.; Staroverov, V. N.; Kobayashi, R.; Normand, J.; Raghavachari, K.; Rendell, A.; Burant, J. C.; Iyengar, S. S.; Tomasi, J.; Cossi, M.; Rega, N.; Millam, N. J.; Klene, M.; Knox, J. E.; Cross, J. B.; Bakken, V.; Adamo, C.; 
Jaramillo, J.; Gomperts, R.; Stratmann, R. E.; Yazyev, O.; Austin, A. J.; Cammi, R.; Pomelli, C.; Ochterski, J. W.; Martin, R. L.; Morokuma, K.; Zakrzewski, V. G.; Voth, G. A.; Salvador, P.; Dannenberg, J. J.; Dapprich, S.; Daniels, A. D.; Farkas, Ö.; Foresman, J. B.; Ortiz, J. V.; Cioslowski, J.; Fox, D. J.; Gaussian Inc.: Wallingford, CT, 2009.

(65) Chai, J.; Head-Gordon, M. Phys. Chem. Chem. Phys. 2008, 10,6615 .

(66) Dunning, T. H. J. Chem. Phys. 1989, 9o, 1007.

(67) Schlegel, H. B. Wiley Interdiscip. Rev.: Comput. Mol. Sci. 2011, 1, 790 .

(68) Singh, U. C.; Kollman, P. A. J. Comp. Chem. 1984, 5, 129.

(69) Besler, B. H.; Merz, K. M.; Kollman, P. A. J. Comp. Chem. 1990, 11, 431.

(70) Petersson, G. A.; Bennett, A.; Tensfeldt, T. G.; Allaham, M. A.; Shirley, W. A.; Mantzaris, J. J. Chem. Phys. 1988, 89, 2193.

(71) Petersson, G. A.; Allaham, M. A. J. Chem. Phys. 1991, 94,6081 .

(72) Cossi, M.; Rega, N.; Scalmani, G.; Barone, V. J. Comp. Chem. 2003, 24, 669.

(73) Barone, V.; Cossi, M. J. Phys. Chem. A, 1998, 102, 1995.

(74) Bismuth, O.; Friedman, N.; Sheves, M.; Ruhman, S. Chem. Phys. 2007, 341, 267.

(75) Mathies, R.; Stryer, L. Proc. Natl. Acad. Sci. U. S. A. 1976, 73, 2169.

(76) Gonzalez-Luque, R.; Garavelli, M.; Bernardi, F.; Merchan, M.; Robb, M.; Olivucci, M. Proc. Natl. Acad. Sci. U. S. A. 200o, 97, 9379 .

(77) Smith, S. O.; Myers, A. B.; Mathies, R. A.; Pardoen, J. A.; Winkel, C.; van den Berg, E. M.; Lugtenburg, J. Biophys. J. $1985,47,653$.

(78) Lee, S. Y.; Heller, E. J. J. Chem. Phys. 1979, 71, 4777.

(79) Grabowski, Z.; Rotkiewicz, K.; Rettig, W. Chem. Rev. 2003, 103, 3899.

(8o) Du, M.; Fleming, G. R. Biophys. Chem. 1993, 48, 101

(81) Haran, G.; Wynne, K.; Xie, A.; He, Q.; Chance, M.; Hochstrasser, R. Chem. Phys. Lett. 1996, 261, 389. 126.

(82) Kandori, H.; Sasabe, H. Chem. Phys. Lett. 1993, 216,

(83) Gozem, S.; Krylov, A. I.; Olivucci, M. J. Chem. Theory Comput. 2013, 9, 284.

(84) Ruhman, S.; Hou, B.; Friedman, N.; Ottolenghi, M.; Sheves, M. J. Am. Chem. Soc. 2002, 124, 8854. 
Table of Contents Figure

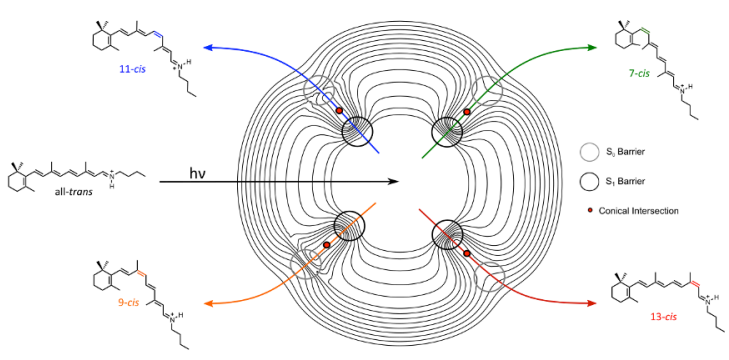


